\title{
Dynamical signatures of Rossby vortices in cavity-hosting disks
}

\author{
C. M. T. Robert ${ }^{1}$, H. Méheut ${ }^{1}$, and F. Ménard ${ }^{2}$ \\ ${ }^{1}$ Université Côte d'Azur, Observatoire de la Côte d'Azur, CNRS, Laboratoire Lagrange, Bd de l'Observatoire, CS 34229, \\ 06304 Nice Cedex 4, France \\ e-mail: clement.robert@oca.eu \\ ${ }^{2}$ Univ. Grenoble Alpes, CNRS, IPAG, 38000 Grenoble, France
}

Received 24 December 2019 / Accepted 13 July 2020

\begin{abstract}
Context. Planets are formed amidst young circumstellar disks of gas and dust. The latter is traced by thermal radiation, where strong asymmetric clumps have been observed in a handful of cases. These dust traps could be key to understanding the early stages of planet formation, when solids grow from micron-size to planetesimals.

Aims. Vortices are among the few known asymmetric dust trapping scenarios. The present work aims to predict their characteristics in a complementary observable. Namely, line-of-sight velocities are well suited to trace the presence of a vortex. Moreover, the dynamics of disks is subject to recent developments.

Methods. Two-dimensional hydro simulations were performed in which a vortex forms at the edge of a gas-depleted region. We derived idealized line-of-sight velocity maps, varying disk temperature and orientation relative to the observer. The signal of interest, as a small perturbation to the dominant axisymmetric component in velocity, may be isolated in observational data using a proxy for the dominant quasi-Keplerian velocity. We propose that the velocity curve on the observational major axis be such a proxy.

Results. Applying our method to the disk around HD 142527 as a study case, we predict that line-of-sight velocities are barely detectable by currently available facilities, depending on disk temperature. We show that corresponding spirals patterns can also be detected with similar spectral resolutions, which will help to test against alternative explanations.
\end{abstract}

Key words. hydrodynamics - instabilities - planets and satellites: formation - protoplanetary disks

\section{Introduction}

Planets are formed in circumstellar disks composed of mainly gas and some solid dust components. Many aspects of the processes implied in their formation remain challenging to explain. More specifically, the transition from small dust grains to large planetesimals face two major obstacles: the drift barrier corresponding to fast inward drifting due to gas headwind, and the collision barrier due to destructive collisions (Chiang \& Youdin 2010). Pressure bumps provide a solution to the drift barrier, as they act as a barrier stopping the drifting solids and forming dust rings. Concentric dusty rings are a common feature in resolved infrared images of protoplanetary disks (Andrews et al. 2018). Pressure bumps are also known to promote the formation of large-scale vortices, through to the Rossby wave instability (RWI), which are proposed as a solution to the barriers in planetesimal formation. Large vortices both stop the dust drift and harness efficient growth by lowering relative speeds between grains. This is why vortices were proposed as a planet-promoting scenario (Barge \& Sommeria 1995; Adams \& Watkins 1995; Tanga et al. 1996; Bracco et al. 1999). Moreover, it is well known that massive planets build up pressure bumps in their vicinity, exciting vortex formation (de Val-Borro et al. 2006, 2007; Fu et al. 2014; Hammer et al. 2017; Andrews et al. 2018; Baruteau et al. 2019), which in turn affects planetary migration (Regály et al. 2013; Ataiee et al. 2014; McNally et al. 2018). The study of large vortices is thus key to understanding planetary formation.

The RWI (Lovelace et al. 1999; Li et al. 2000, 2001) is a promising vortex-forming scenario, and is expected where sharp density gradients are found. So-called transitional disks provide such conditions at the outer edge of large ( $\sim 5-100 \mathrm{AU})$ gas cavities they host. Extensive computational effort has been dedicated to studying the long-term evolution of RWI vortices (Fu et al. 2014; Méheut et al. 2012a; Regály \& Vorobyov 2017a; Andrews et al. 2018). Overall, eddies tend to form in a few tenths of orbital periods and survive for $10^{3}$ to $10^{4}$ orbital periods.

Concurrently, asymmetric dust crescents are being observed in thermal radiation of a growing number of targets (Cazzoletti et al. 2018; Dong et al. 2018; Isella et al. 2018; Casassus et al. 2019; Pineda et al. 2019) as well as in scattered emission (Benisty et al. 2018). Those clumps are candidates for large vortices, and there have been attempts to explain their formation as vortexdriven (Regály et al. 2012; Birnstiel et al. 2013). Alternatively, disk eccentricity (Ataiee et al. 2013) and excitation by an eccentric companion (Astropy Collaboration 2018) were proposed to explain this azimuthal dust excess, however not reproducing the observed dust-to-gas ratio.

Complementary measurements of the gas dynamics would be of great help in constraining and rejecting concurrent explanations. Continuum emission traces the spatial distribution of dust grains dynamically coupled with the gas, so it provides indirect information concerning the underlying gas dynamics. However, direct measurements of the gas radial velocity can now be achieved through Doppler-shifting of molecular lines, as a result of increasingly sophisticated data reduction techniques (Yen et al. 2016; Teague et al. 2016; Teague \& ForemanMackey 2018) and ever-enhanced spatial resolution (Andrews et al. 2018). It is becoming possible to use these data to build connections to continuum asymmetries (Casassus et al. 2015a; Casassus \& Pérez 2019) or to search for planet-induced 
deviations (Pinte et al. 2018, 2019; Teague et al. 2018a; Pérez et al. 2020).

Hence, observations in molecular line emission are essential to confirm or reject current and future vortex candidates. The present paper aims to characterize the dynamical signatures expected for a single large Rossby eddy forming in the inner rim of a cavity, by the means of hydro simulations.

The paper is organized as follows. First, we describe the numerical setup of our hydro simulations in Sect. 2. We then provide insight into the observability of resulting vortices and propose a method to extract their signature from observational data in Sect. 3. Finally, we discuss the limits of our approach in Sect. 4 and conclude in Sect. 5.

\section{Hydro simulations setup}

We perform 2D hydro simulations via MPI-AMRVAC 2.2 (Porth et al. 2014; Xia et al. 2018). Namely, we solved Euler equations for an inviscid gas as follows:

$\partial_{t} \Sigma+\nabla \cdot(\Sigma v)=0$

$\left(\partial_{t}+\boldsymbol{v} \cdot \boldsymbol{\nabla}\right) \Sigma \boldsymbol{v}=-\Sigma \boldsymbol{\nabla} \phi-\nabla p$,

where $\Sigma$ and $\boldsymbol{v}$ stand for surface density and velocity, respectively, $\phi \propto-1 / r$ is a central gravitational potential, and $p$ is the vertically integrated pressure. The latter is prescribed by a barotropic equation of state $p=S \Sigma^{\gamma}$, where $S=86.4$ (code units ${ }^{1}$ ) characterizes the entropy and $\gamma=5 / 3$ is the adiabatic index. Sound speed is given as $c_{\mathrm{s}}^{2}=\gamma p / \Sigma$. Equations are solved on a linearly spaced polar grid $(r, \varphi)$ with a fixed resolution $(512,512)$, ranging from $r_{\min }=75 \mathrm{AU}$ to $r_{\max }=450 \mathrm{AU}$ and $\varphi \in[0,2 \pi]$. Numerical convergence was checked against runs with double resolution in each direction. The MPI-AMRVAC 2.2 simulations use finite-volumes Reimann solvers. A two-step hllc integration scheme (Harten et al. 1983) and a Koren slope limiter (Koren 1993) are used in our simulations.

The model is physically inviscid. The numerical viscosity, expressed in terms of the widely used " $\alpha$ " paradigm (Shakura \& Sunyaev 1973), was estimated to lie between $2 \times 10^{-8} \leq$ and $\leq$ $3 \times 10^{-4}$ in the vortex-forming region. Details on this estimations are given in Appendix B.

The disk is truly "massless" in that both self-gravity and indirect terms due to the motion of barycenter are neglected in the computation of the gravitational potential. Zhu \& Baruteau (2016) show that including either or both of these contributions affects the evolution of the vortex. In particular, the inclusion of indirect terms promotes a radial displacement of the structure and overall increases the density contrast with respect to its background. This latter result was also confirmed by Regály \& Vorobyov (2017b) for vortices formed in a viscosity transition region. Because of these combined effects, the velocity of the structure is also modified, while a direct comparison is nontrivial.

\subsection{Initial conditions}

The initial gas surface density features a smooth radial density jump, modeling a disk cavity as

$\Sigma(r, t=0)=\Sigma_{0}\left(r / r_{*}\right)^{-1} \times \frac{1}{2}\left[1+\tanh \frac{r-r_{\mathrm{j}}}{\sigma_{\mathrm{j}}}\right]$,

1 Our code unit system is completely described by mass, length and time normalisation constants respectively $m_{*}=1$ solar mass, $r_{*}=$ $100 \mathrm{AU}$ and $t_{*}=1$ orbital period of a test particle at $r=r_{j}$.

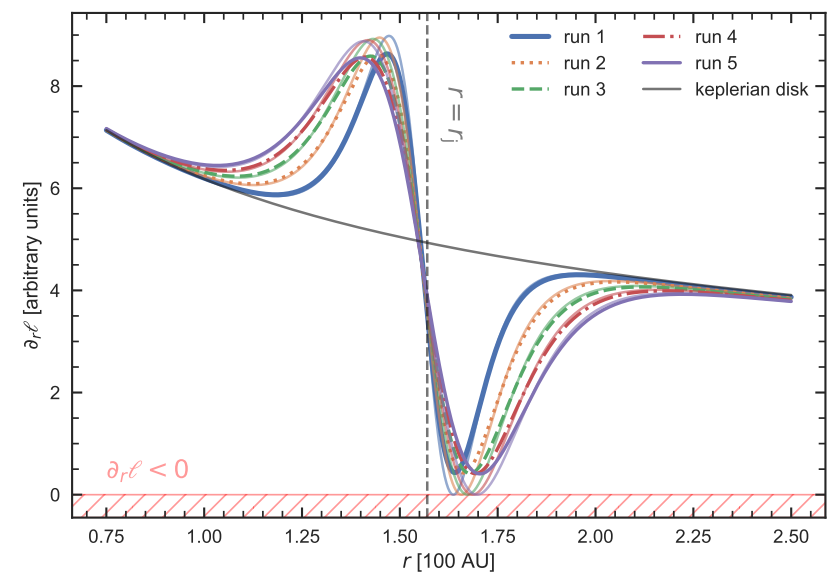

Fig. 1. Initial gradient in specific angular momentum $\ell$ for our 5 simulations (thick lines, ranked from coldest to hottest) compared to the Keplerian case. The width of the density "jump" region $\sigma_{\mathrm{j}}$ is adjusted in two steps. First, the critical value $\sigma_{\mathrm{j}}^{\text {crit }}$ for which the global minimum in $\partial_{r} \ell$ is exactly 0 (thin solid lines) is derived. Any value $\sigma_{\mathrm{j}}<\sigma_{\mathrm{j}}^{\text {crit }}$ would give rise to a rotationally unstable region, characterized by $\partial_{r} \ell<0$. An arbitrary $5 \%$ margin was applied $\sigma_{\mathrm{j}}=1.05 \times \sigma_{\mathrm{j}}^{\text {crit }}$ in our runs (thick dashed lines).

where $r_{\mathrm{j}}$ and $\sigma_{\mathrm{j}}$ are the radial location and the width of the jump respectively, and $r_{*}=100 \mathrm{AU}$ is a normalization factor. The initial equilibrium azimuthal velocity is defined as

$$
\frac{v_{\varphi}^{2}}{r}=\frac{G M}{r^{2}}+\frac{\partial_{r} p}{\Sigma}
$$

where $G$ is the universal gravity constant and $M$ is the mass of the central star.

Observational constraints for HD 142527 are used to tune numerical values, wherever applicable, as we now detail. We assume $M=2.2 M_{\odot}$, compatible with existing estimations (Verhoeff et al. 2011; Casassus et al. 2015b). We choose a standard radial density slope in $r^{-1}$, which is also compatible with estimate from Verhoeff et al. (2011) in the optically thin approximation at $1 \mathrm{~mm}$. Distance to star is now known with good precision $157 \pm{ }_{6}^{7}$ pc thanks to Gaia Collaboration (2016), which implies the cavity lies at $r_{\mathrm{j}}=157 \mathrm{AU}$ for an angular size of $1{ }^{\prime \prime}$. 0 (Casassus et al. 2012). The reference setup has an aspect ratio, or "temperature" $h \equiv H\left(r_{\mathrm{j}}\right) / r_{\mathrm{j}} \simeq 0.09$, where $H(r)$ is the disk scale height.

Other simulations with $h \in[0.09 ; 0.16]$ were performed, and labeled run 1 to run 5 by increasing value in $h$. These are discussed in Sect. 3.3. The derivation of this parameter is detailed in Appendix A.1. As this temperature is varied, we adjust the density jump's width $\sigma_{\mathrm{j}}$ within $5 \%$ of its critical value, where the disk becomes rotationally unstable under Rayleigh's criterion (Rayleigh 1879). Doing so, we approach the physical upper limit in vortex velocity after the RWI saturates. The corresponding signature in the specific angular momentum $\ell=r v_{\varphi}$ is illustrated in Fig. 1. The computed values for $\sigma_{\mathrm{j}}$, and for runs from 1 to 5, are $[11.6,14.7,16.9,18.7,20.2]$ AU. Even in the hottest case, the simulation box extends at least $4 \sigma_{\mathrm{j}}$ away from the density jump center $r_{\mathrm{j}}$. Unless explicitly stated, all figures show the results for the reference model.

\footnotetext{
2 The vertical spreading of the disk is physically caused by heating and usually characterized by a scale height.
} 

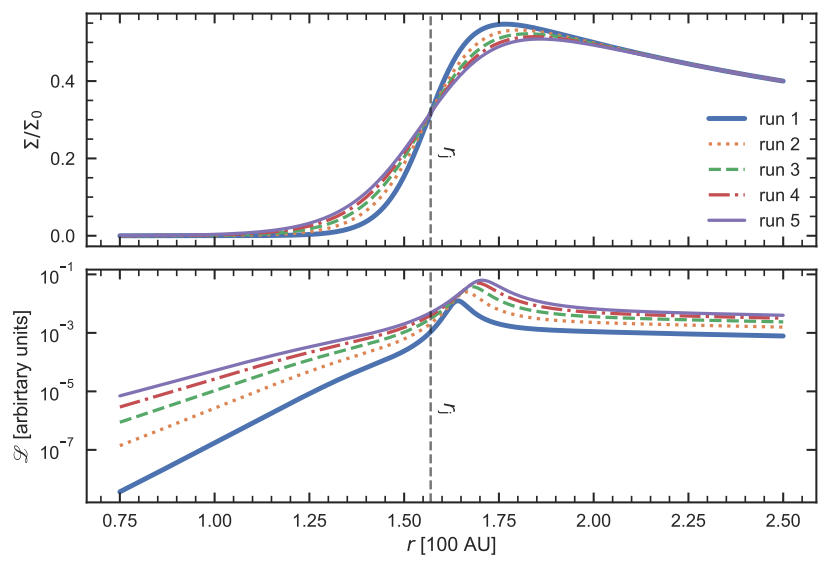

Fig. 2. Initial radial profiles in surface density (top) and the $\mathscr{L}(r)$ function (defined in Eq. (5)) for our 5 simulations. In each model, $\mathscr{L}(r)$ features a clear local maximum, which is a necessary condition to RWI growth.

\subsection{Boundary conditions}

Boundary conditions are imposed through ghost cells outside of the domain and wave-killing region in the active domain. In the radial direction, ghost cells are fixed to the initial equilibrium values for density and azimuthal momentum. The radial momentum is copied from the first cells to the ghost cells at the inner boundary, and extrapolated linearly with no-inflow condition at the outer edge. However, these boundary conditions have low impact as standard damping zones (de Val-Borro et al. 2006) are also used to avoid reflections at domain edges. The domain is periodic in the azimuthal direction.

\subsection{Rossby wave instability and vortex formation}

Rossby wave instability is similar to the Kelvin-Helmholtz instability in a differentially rotating Keplerian disk. The instability tends to convert excess shear into vorticity. Lovelace et al. (1999) showed that a local extremum in the background potential vorticity is a necessary condition to RWI. More recent works have clarified that a minimum is required (Lai \& Tsang 2009; Ono et al. 2016). The key function is defined as

$\mathscr{L}(r)=\frac{1}{2} \frac{\Sigma}{(\nabla \times \boldsymbol{v}) \cdot \boldsymbol{e}_{z}} S^{2 / \gamma}$.

We exhibit this key function within our initial setup in Fig. 2, showing the existence of a local maximum in $\mathscr{L}(r)$, corresponding to a minimum in vorticity.

We find that, in order to excite the RWI unstable modes, it is useful to add random perturbations. We chose to perturb the radial velocity, which is zero otherwise, as

$v_{r}(r, \varphi, t=0)=c_{\mathrm{s}} \psi(r, \varphi) \exp \frac{-\left(r-r_{\mathrm{j}}\right)^{2}}{2 \sigma_{\mathrm{j}}^{2}}$,

where $\psi(r, \varphi) \in\left[-10^{-2}, 10^{-2}\right]$ is a uniformly distributed random variable drawn for each grid cell. After the instability has saturated, we obtain a single vortex shown in Fig. 3. In a frame that is co-rotating with the vortex, its global structure is quasistationary as shown in Fig. 4. The radial density profile at the azimuth of the density maximum is plotted at different times. The orange dotted curve at $t=10$ features the most noticeable

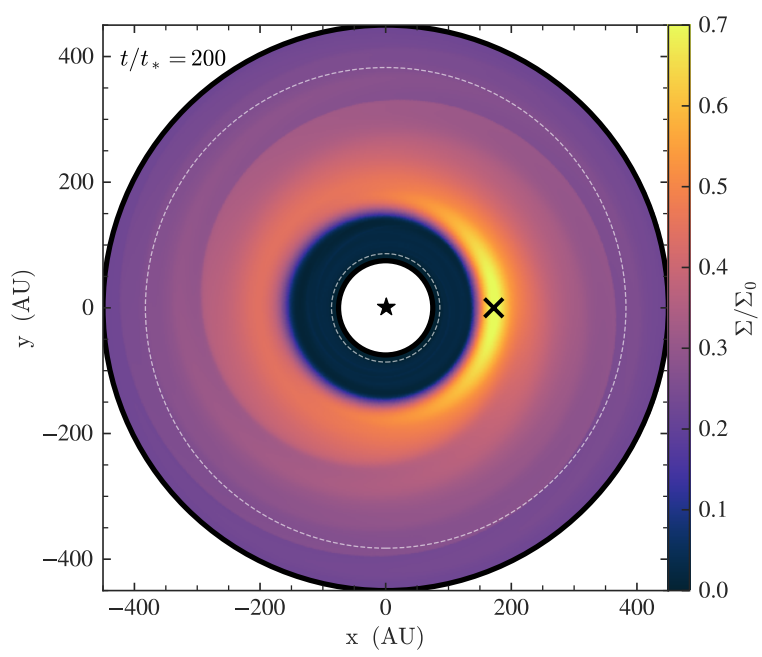

Fig. 3. Gas surface density plotted in Cartesian coordinates $(x, y)$ after $t=200$ orbital periods $\left(t_{*}\right)$. The global density maximum is indicated by a black cross. The position of the central star is denoted as a " $\star$ " symbol. The simulation box radial limits are drawn as solid black circles, while the dashed-line circles indicate the limits of wave damping zones.
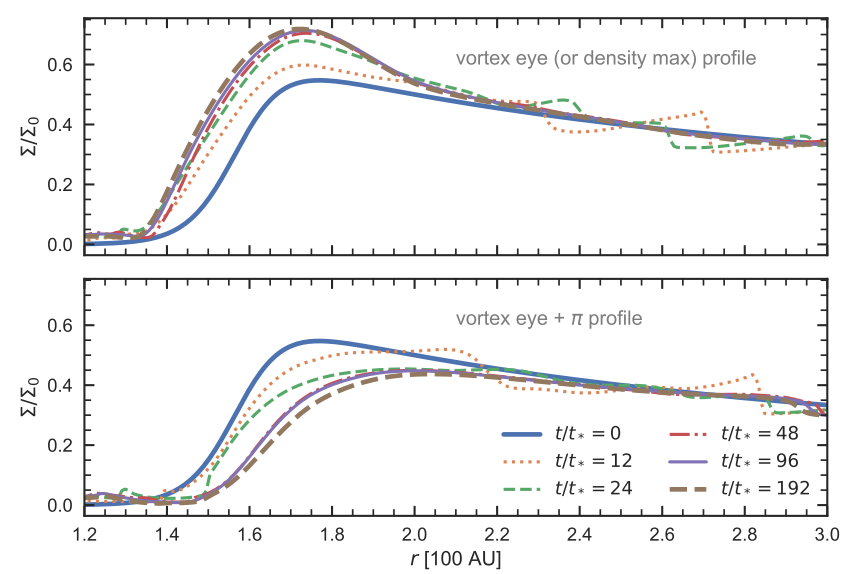

Fig. 4. Time evolution of the radial density profile, plotted as slices at the azimuth of the density maximum where the vortex eye lies (top) and its radial opposite (bottom). The slices correspond to the $y=0$ axis in Fig. 3, with $x>0$ (top) and $x<0$ (bottom), respectively. After $\sim 40$ orbital periods, the disk has practically reached a stationary state. The cavity profile itself has become uneven, showing a non-zero eccentricity.

fluctuations, as smaller eddies are still undergoing a merger and strong spiral waves are launched outward. After 40 orbital periods, the surface density of the vortex is stabilized and does not rapidly evolve any more. Thus we consider this state as quasistationary; we take a look at the dynamics of the structure in the next section.

\section{Vortex signatures in dynamics}

In this section, we provide observational signatures obtained from the dynamics of the vortex. The observable studied in this work is the velocity projected along the line of sight $v_{\mathrm{LOS}}$. We first discuss an adequate decomposition of the velocity field to characterize the signatures. We then study their observability against disk orientation and provide insight into how disk aspect ratio affects observed velocities. 


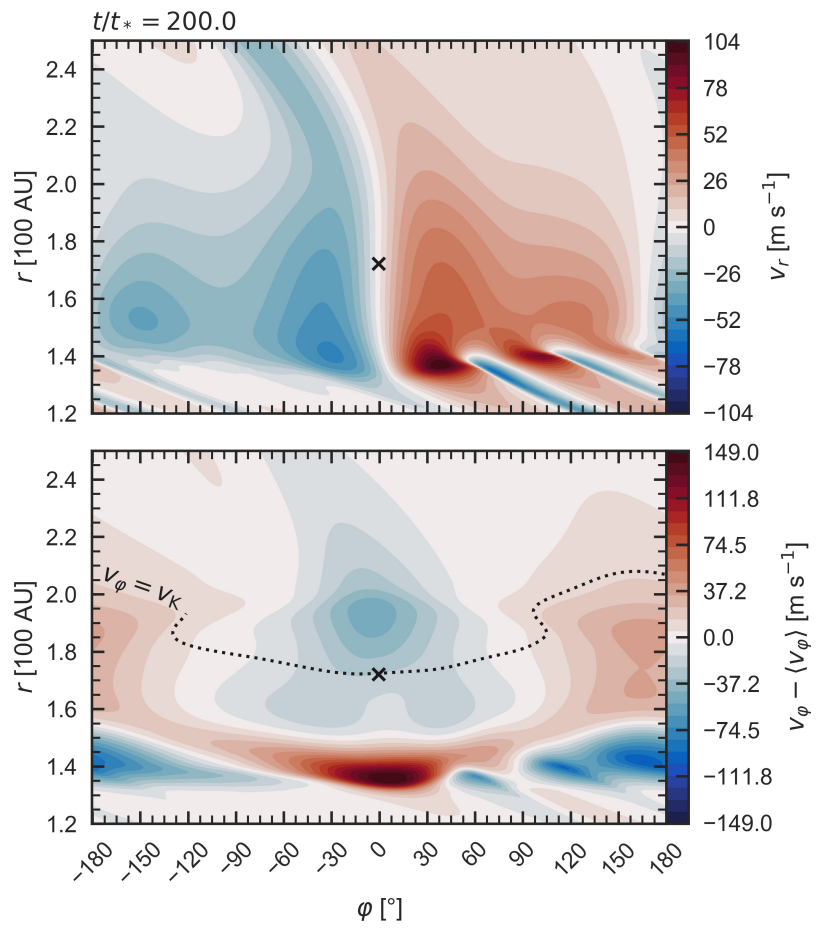

Fig. 5. Polar components of a the velocity field of a vortex. Top: radial velocity. Bottom: azimuthal velocity, where the axisymmetric part $\left\langle v_{\varphi}\right\rangle$ is carried out. The pressure maximum is indicated by a black cross. The snapshot is taken at $t=200$ orbital periods. The dotted line in the bottom panel indicates fluid in exact Keplerian rotation.

\subsection{Extracting dynamical signatures}

The dynamics of a disk is dominated by rotation around the central star. In an axisymmetric stationary state, the net radial force is zero, as in Eq. (4). Owing to pressure gradients, the radial equilibrium slightly departs from Keplerian motion. This is the sub-Keplerian rotation in a disk with negative radial pressure gradient. As a dynamical structure, a vortex exposes little difference to global rotation. Thus, it is useful to decompose the angular velocity $v_{\varphi}$ as

$v_{\varphi}=\left\langle v_{\varphi}\right\rangle+\left(v_{\varphi}-\left\langle v_{\varphi}\right\rangle\right) \equiv\left\langle v_{\varphi}\right\rangle+v_{\varphi}^{\prime}$,

where $\langle\cdot\rangle$ is the azimuthal average operator, and we denote the non-axisymmetric part as $v_{\varphi}^{\prime}$. Hence the total velocity field $\boldsymbol{v}$ can be decomposed in the polar basis $\left(\boldsymbol{e}_{r}, \boldsymbol{e}_{\varphi}\right)$ as

$\boldsymbol{v}=v_{r} \boldsymbol{e}_{r}+\left(\left\langle v_{\varphi}\right\rangle+v_{\varphi}^{\prime}\right) \boldsymbol{e}_{\varphi} \equiv\left\langle v_{\varphi}\right\rangle \boldsymbol{e}_{\varphi}+\boldsymbol{v}^{\prime}$

In the absence of a global accretion flow, there is no relevant axisymmetric part in $v_{r}$. Hence we consider that dynamical signatures of non-axisymmetric features reside in $\boldsymbol{v}^{\prime}=v_{r} \boldsymbol{e}_{r}+v_{\varphi}^{\prime} \boldsymbol{e}_{\varphi}$. Both components of this residual radial and azimuthal velocity are quantified in Fig. 5 and are of similar amplitudes. For comparison, the typical Keplerian speed at the vortex position $(r \sim 180 \mathrm{AU})$ is $v_{\mathrm{K}}=3.3 \mathrm{~km} \mathrm{~s}^{-1}$, that is, one to two orders of magnitudes larger than the deviation due to the vortex, and one order of magnitude larger than the local sound-speed $c_{\mathrm{s}}$. The amplitude in the azimuthal velocity is as high as $300 \mathrm{~m} \mathrm{~s}^{-1}$ for this reference (coldest) model. This sets a first upper limit to the spectral resolution required for a direct detection to about $100 \mathrm{~m} \mathrm{~s}^{-1}$. This is achievable for bright lines with the Atacama Large Millimeter/submillimeter Array (ALMA), for example,
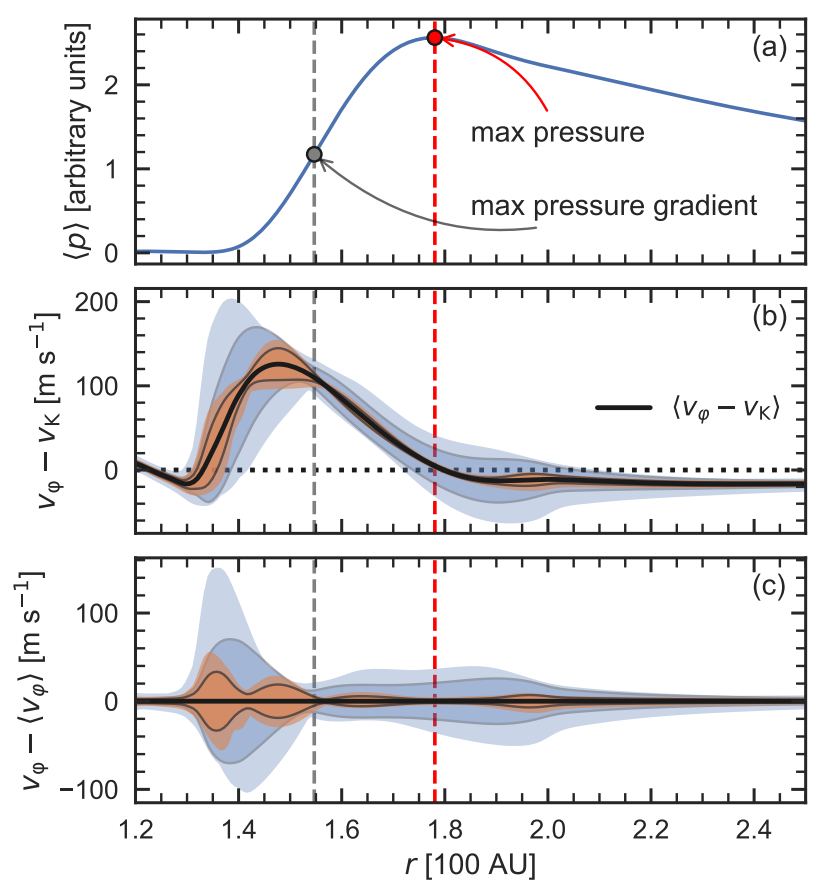

Fig. 6. Comparison between Keplerian velocity $v_{\mathrm{K}}$ and average azimuthal velocity $\left\langle v_{\varphi}\right\rangle$ as 1D masks. (a) Pressure profile in arbitrary units. ( $b$ and $c$ ) Azimuthal velocity cross-sections, with offsets (masks) $v_{\mathrm{K}}$ and $\left\langle v_{\varphi}\right\rangle$, respectively. The average $\langle V-$ mask $\rangle$ is indicated by a thick black line, while shadows show the amplitude and standard deviations in blue $\left(V=v_{\varphi}\right)$, and orange $\left(V=\frac{1}{2}\left(v_{\varphi \text {,left }}+v_{\varphi \text {,right }}\right)\right)$. Data are taken at $t=200$ orbital periods (inspiration for this figure was drawn from Teague et al. 2018a).

for the $\mathrm{CO}(2-1)$ or the $\mathrm{CO}$ (3-2) transitions, using channel widths of 70 or $120 \mathrm{kHz}$ (or narrower), respectively. For example, van der Marel et al. (2016) successfully detected the ${ }^{13} \mathrm{CO}(3-2)$ and $\mathrm{C}^{18} \mathrm{O}$ (3-2) lines of SR21, HD 135344B, DoAr44, and IRS 48 with good signal-to-noise ratio $(\mathrm{S} / \mathrm{N})$ (peak $\mathrm{S} / \mathrm{N}$ in the integrated intensity map up to 30 for the ${ }^{13} \mathrm{CO}$ line) with spectral resolution of $0.1 \mathrm{~km} \mathrm{~s}^{-1}$ and angular resolution of 0.25 . Boehler et al. (2017) obtained data with similar angular and spectral resolutions for HD 142527 but with much higher S/N. All sources are well detected in the lines; increasing the spectral resolution by another factor of 2 , as well as the $\mathrm{S} / \mathrm{N}$, is possible within a reasonable amount of time $(<12 \mathrm{~h})$. We note that there is a non-zero azimuthal velocity deviation at the maximum density/pressure (i.e. $v_{\varphi}^{\prime} \neq 0$ ), as seen in Fig. 5. Because the vortex is an asymmetric structure, the radial position of the pressure extremum varies with the azimuth. Consequently, the line of exact Keplerian rotation is not circular as shown in Fig. 5.

However, the decomposition proposed in Eq. (8) is vain unless the proposed dominant term $\left\langle v_{\varphi}\right\rangle$ can be subtracted from observations. While a Keplerian fit is usually a suiting approximation of the dominant velocity term, it proves insufficient near sharp density jumps. As shown in Figs. 6a and b, subtracting a Keplerian power law leaves systematic velocities caused by pressure gradients. In the density transition region, those systematics dominate over the variability in the remaining signal.

However, we further show (Figs. $6 \mathrm{~b}$ and c) that averaging two facing cross sections in azimuthal velocities consistently yields a much better approximation for the global azimuthal average $\left\langle v_{\varphi}\right\rangle$, with a standard deviation $\leq 20 \mathrm{~m} \mathrm{~s}^{-1}$. This is a direct sign that the non-axisymmetric parts of the azimuthal velocities $v_{\varphi}^{\prime}$ in opposing disk halves are anticorrelated, although not strictly equal in 


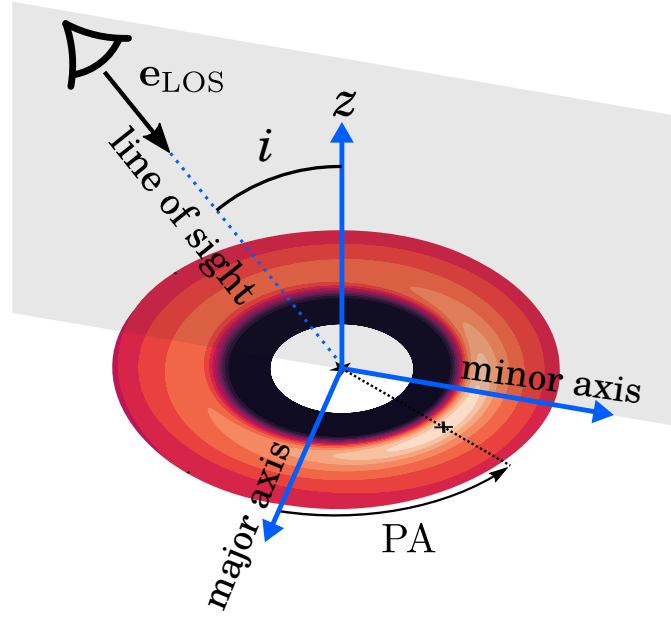

Fig. 7. Sketch view of our notations. The gray shadow shows the plane containing the vertical $\boldsymbol{e}_{z}$ and the line of sight.

amplitudes. Given that detection is only sensitive to azimuthal velocities on the observational major axis $x$ of the disk, we naturally obtain a satisfying method to subtract $\left\langle v_{\varphi}\right\rangle$ from the whole signal. Consequently, we now confidently assume that the axisymmetric part $\left\langle v_{\varphi}\right\rangle$ of the azimuthal velocity can indeed be removed with good precision from observations, and we only consider the remaining components of $\boldsymbol{v}^{\prime}$ exhibited in Fig. 5.

\subsection{Vortex detection in line-of-sight velocities}

Gas velocity is usually detected through Doppler-shifting in molecular lines. It is therefore the velocity component parallel to the line of sight that is probed. Within optically thick lines, the resulting velocity profile can be blurred by vertical integration over disk height. It is beyond the scope of the present work to investigate this second-order effect, so we neglect both optical and geometrical thickness effects. This approach is reasonable within the assumption that emissive molecular regions are geometrically thin and well resolved (as remarked by Teague et al. 2018b). Furthermore, full 3D simulations showed that, in a stationary state, a vortex tends to be tubular and that its vertical velocity is negligible (Lin 2012; Zhu \& Stone 2014; Richard et al. 2013). This comforts us in the idea that, for long-lived vortices, it is reasonable to ignore this component. This also means we ignore the vertical extension in the conical shape of the emissive layer. However, it can easily be shown that for inclinations lowers than $45^{\circ}$, even a very high emissive layer $z \simeq 5 H$ and a large aspect ratio $H / r=0.2$, can in principle be deprojected as long as it remains spatially thin.

To study vortex dynamical signatures, we use here cylindrical coordinates centered on the star. The radial axis $(\varphi=0)$ is the observational major axis, and the upper part of the disk $(z>0)$ is defined to be the one seen by the observer Fig. 7. Thus, the lineof-sight direction $\boldsymbol{e}_{\mathrm{LOS}}(i, \varphi)$, defined as pointing away from the observer, can be written in the disk cylindrical basis $\left(\boldsymbol{e}_{r}, \boldsymbol{e}_{\varphi}, \boldsymbol{e}_{z}\right)$ as

$\boldsymbol{e}_{\mathrm{LOS}}(i, \varphi)=-\left(\sin (i) \cos (\varphi) \boldsymbol{e}_{r}+\sin (i) \sin (\varphi) \boldsymbol{e}_{\varphi}+\cos (i) \boldsymbol{e}_{z}\right)$,

and it follows that the line-of-sight velocity corresponding to $\boldsymbol{v}^{\prime}$ is written as

$v_{\mathrm{LOS}}^{\prime} \equiv \boldsymbol{v}^{\prime} \cdot \boldsymbol{e}_{\mathrm{LOS}}=-\sin (i)\left(\sin (\varphi) v_{r}+\cos (\varphi) v_{\varphi}^{\prime}\right)$.
Hence, the effective observable $v_{\mathrm{LOS}}^{\prime}$ mixes $v_{r}$ and $v_{\varphi}$. For a 2D vortex disk inclination equally affects all projected velocities and only acts as a scaling factor $\sin (i)$. An inclination $i=27^{\circ}$, corresponding to the estimated value for HD 142527 (Fukagawa et al. 2013), is used in the following applications. This choice is arbitrary and used as a textbook case. We note that this inclination is moderate. Deprojection would still be feasible at up to $i=45^{\circ}$, where projected velocities would be 1.5 times larger, making detection easier. Figure 8 shows the morphology of the observable $v_{\text {LOS }}^{\prime}$ (large panels), along with corresponding components $v_{r}$ and $v_{\varphi}^{\prime}$ (small panels), for four different values of PA. This result constitutes an idealized case, built on the assumption that the axisymmetric component $\left\langle v_{\varphi}\right\rangle$ can be exactly subtracted from observational data. We note that we are set in the particular case where the PA rotates in the same direction as the disk. When not so, sign in $v_{\text {LOS }}^{\prime}$ must simply be inverted. At all position angles (PAs), the anticyclonic motion of the vortex around the density maximum is apparent in $v_{\mathrm{LOS}}^{\prime}$. This point roughly coincides with the maximum luminosity at most wavelengths, and can be located within continuum observations, if not directly in molecular lines used to infer projected velocities.

We note that the eye of the vortex and the region immediately facing it have similar Doppler shifts (e.g., both blue at $\left.\mathrm{PA}=0^{\circ}\right)$. This is an expected outcome of subtracting the azimuthally averaged velocity, since the both regions are local extrema along the azimuthal direction. Another signature of the vortex is the azimuthal proximity between the maximum density (black cross) and the projected velocity extrema. The latter two points are determined by the physical on-site velocity as well as the inclination of the system, and hence are virtual positions. Their physical separation is maximized for $\mathrm{PA}=90^{\circ}$ and minimized for $\mathrm{PA}=0^{\circ}$. A direct implication is that detecting a vortex lying on the major axis requires greater angular resolution. However, little dependence of the velocity range on the PA is found. The topography of the signal changes with the PA but the anticyclonic region stands out regardless the orientation. The signature is also typical with a sign reversal in the vicinity of the pressure maximum, along the major axis direction. This characteristic behavior, sign change, is easier to measure in relative than the absolute small velocities and would be observed even with a beam covering the vortex almost entirely (about $100 \mathrm{AU}$, or 0.' 6 in the case of HD 142527).

\subsection{Detectability against disk temperature}

Although our setup is constrained by observations, its temperature (or equivalently $h$ ) is not. Indeed the temperature gives the sound speed, which is fundamental to estimate the vortex velocity. To study this dependence, four additional simulations with higher temperatures $(h \in[0.094,0.119,0.136,0.150,0.161])$ were performed. In Fig. 9, we show contours of projected velocity $v_{\mathrm{LOS}}^{\prime}$, sampled at an interval corresponding to a tenth of the obtained dynamical range, namely $10 \mathrm{~m} \mathrm{~s}^{-1}$. The reference, "coldest" setup produces the lowest velocities ranging from -20 to $+20 \mathrm{~m} \mathrm{~s}^{-1}$, where most of the "detected" structure is within the vortex region. The direct observation of a peak-to-valley velocity shift of about $40 \mathrm{~m} \mathrm{~s}^{-1}$ is challenging but is within reach of ALMA. Boehler et al. (2017) observed HD 142527 for a total of four hours during Cycle 1, targeting the continuum and ${ }^{13} \mathrm{CO}(3-$ 2) and $\mathrm{C}^{18} \mathrm{O}(3-2)$ lines with a spectral resolution of $110 \mathrm{~m} \mathrm{~s}^{-1}$ (after Hanning smoothing). The disk is detected in both lines at high $\mathrm{S} / \mathrm{N}$. The angular resolution of the observations was $45 \mathrm{AU}$ (beam $0.27 \times 0.31$ ). The presence of a velocity signature is currently being investigated in that data set (Boehler et al., in prep.). 

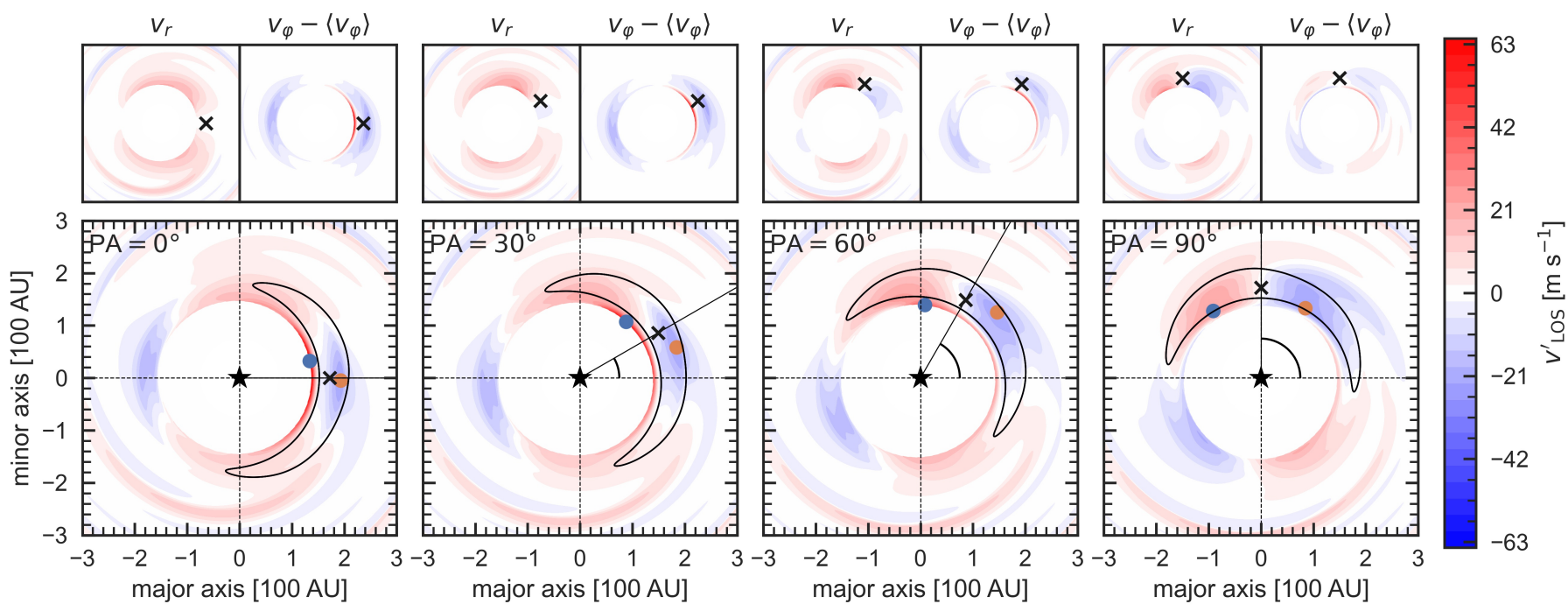

Fig. 8. Line-of-sight velocities (bottom large panels) as defined in Eq. (10), applied to HD 142527 with $i=27^{\circ}$, and varying PA. Top panels: exhibit the corresponding polar components. Color reflects implied Doppler-shifts in molecular lines. Blue/orange dots indicate extreme values in $v_{\mathrm{LOS}}^{\prime}$. Leftmost panel: vortex's spatial extension is shown as a solid contour which corresponds to $\Sigma=0.5 \Sigma_{0}$, where $\Sigma_{0}$ is the scaling factor used in Eq. (3). The inner cavity, where fast spiral waves are launched but surface density is low, is not shown here : regions with $\Sigma / \Sigma_{0}<0.1$ are masked. As a proxy for the vortex's eye, a black cross indicates the density maximum.

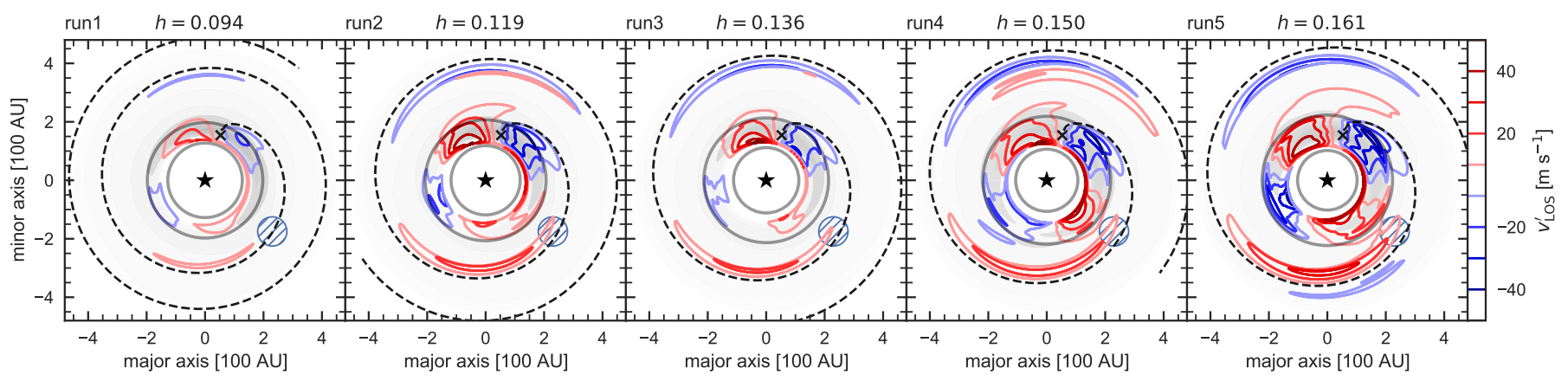

Fig. 9. Comparative view of $v_{\mathrm{LOS}}^{\prime}$ with varying disk temperature $h$. Disk orientation is taken consistent with values found in the literature on HD $142527\left(i=27^{\circ}\right.$ Fukagawa et al. 2013, PA $=71^{\circ}$ Kataoka et al. 2016). A mock-up of a spectral resolution of $12.5 \mathrm{~m} \mathrm{~s}^{-1}$ is shown. The reference setup occupies the leftmost panel. Velocities in the cavity are masked as in Fig. 8. In dashed lines, the best fit spirals following (Huang et al. 2019; Eq. (2) therein) are overplotted, based on linear perturbation theory (Goldreich \& Tremaine 1979; Rafikov 2002; Muto et al. 2012). Those fits were computed using $v_{r}=0$ contours as input data. As a visual indicator, surface density is underplotted in grayscale. Additionally, gray circles indicate the $3 \sigma_{\mathrm{j}}$ region around the vortex eye, which is used later in Fig. 10. As in other figures, the black cross indicates the density maximum.

This angular resolution is sufficient to resolve the vortex in HD 142527 and the spectral resolution can be improved by a factor of 2 on the brighter ${ }^{12} \mathrm{CO}$ line (Perez et al. 2015), or on the ${ }^{13} \mathrm{CO}$ and $\mathrm{C}^{18} \mathrm{O}$ lines by increasing the time spent on-source.

At higher temperatures, more structure is revealed as the spiral arm unravels. Figure 10 shows the $v_{\text {LOS }}^{\prime}$ variation amplitude, against temperature (left panel) and time (right panel). Although the upper bound of this range consistently increases with temperature, we note that the mean value is almost unchanged from run 4 to run 5. Indeed, in runs 3 to 5, the amplitude of time variations are significantly higher than for the reference run. These large variations are related to the life cycle of a secondary spiral arm that appears in hot cases, as illustrated in Fig. 11. However, because this secondary spiral is most prominent when the disk itself becomes visibly eccentric, it is likely that this structure would be affected if the indirect gravitational terms were included in the model. A conservative conclusion is that only the lower boundary of the variation interval should be taken into account. Additionally, we observe that between runs 4 and 5, the dynamical range stagnates at $94 \mathrm{~m} \mathrm{~s}^{-1}$. Indeed, this is the range shown in Fig. 9, where the simulations are shown at a time $\left(t / t_{*}=200\right)$ that minimizes its amplitude. This saturation is likely caused by the instability in the eccentricity of the cavity, thus we infer that validity of massless disk models is disputable in the hottest case (run 5). We note that a previous study of the gas dynamics in HD 142527 (Casassus et al. 2015b) did not provide evidence of any strong asymmetric structure. This may indeed be due to a lack of spectral resolution $\left(\sim 1 \mathrm{~km} \mathrm{~s}^{-1}\right)$.

\subsection{RWI spirals}

Spirals structures are detected in HD 142527 (Fukagawa et al. 2006; Casassus et al. 2012; Rameau et al. 2012; Avenhaus et al. 2014; Christiaens et al. 2014). Several scenarios have been proposed to understand their origin, such as self-gravitational instability (SGI), excitation by the stellar companion (Biller et al. 2012; Astropy Collaboration 2018), connection to a shadow cast by a misaligned inner disk (Montesinos et al. 2016), or a combination of several effects (Christiaens et al. 2014). Spirals are also a natural outcome of RWI, as Rossby waves are coupled to spiral 

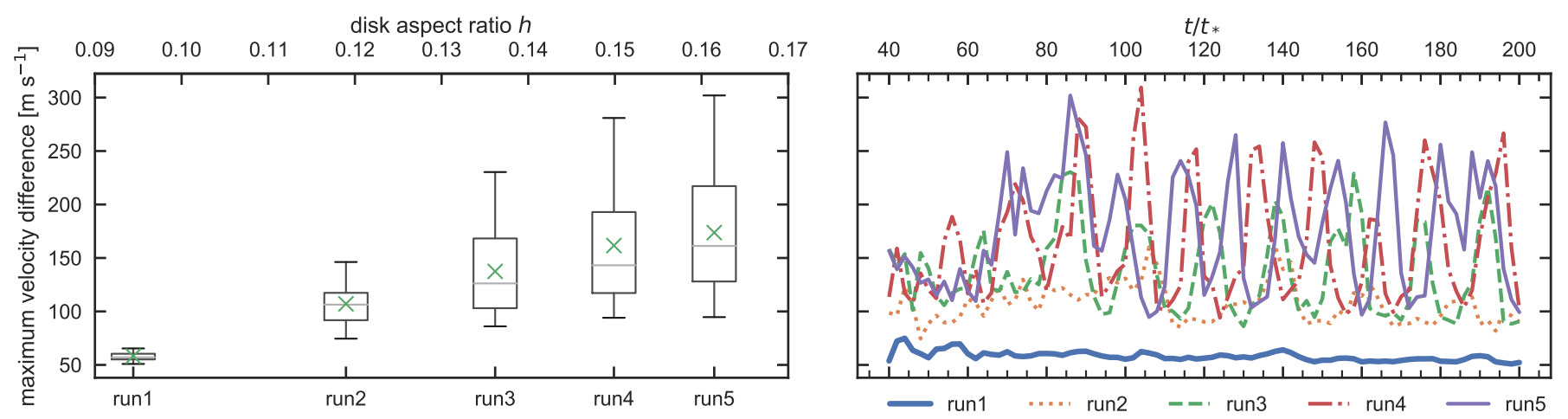

Fig. 10. Amplitude in $v_{\text {Los }}^{\prime}$ across the annular region shown in Fig. 9, throughout the simulation time, represented as a boxplot (left). The whole time series is unraveled in the right panel. It is sampled every 2 orbital periods (the output rate of our simulations). Although the first run is remarkably constant, runs 3 to 5 exhibit significant dispersion within this metric, while the mean value (green crosses) itself is stabilized. The period of these oscillations corresponds to the life cycle of a secondary spiral arm, illustrated in Fig. 11.

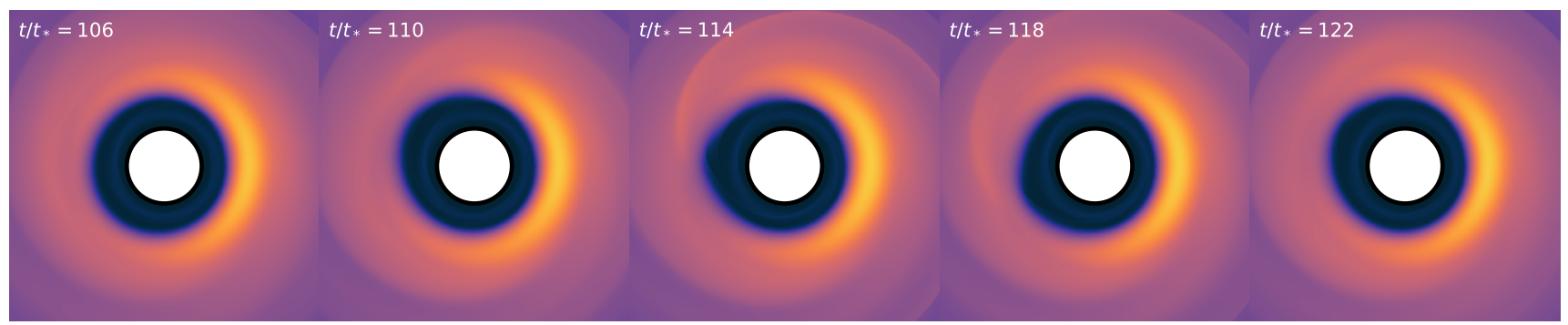

Fig. 11. Formation/dissipation cycle of a secondary spiral arm connected with disk eccentricity, illustrated for the most prominent case, run 5. Color maps density (same scale as Fig. 3). This secondary spiral is a transient and periodic phenomenon, which is responsible for large oscillations in maximum projected velocity as measured in Fig. 10
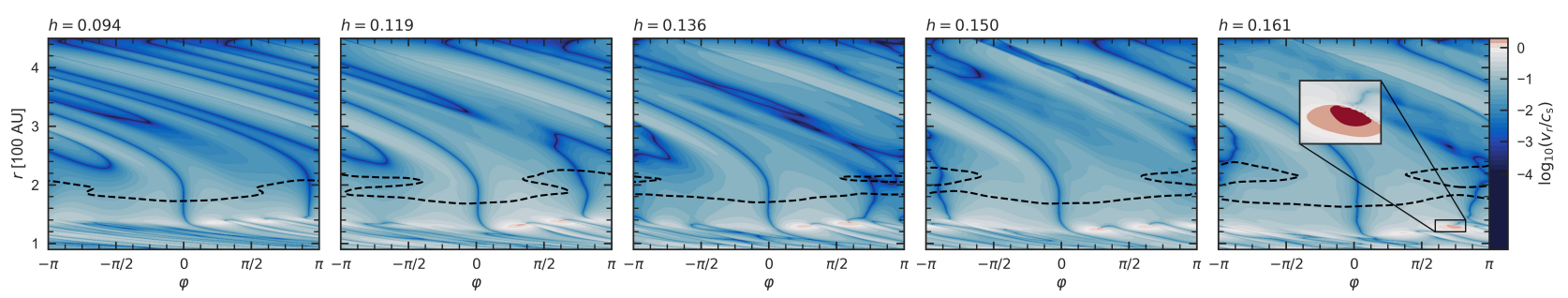

Fig. 12. Radial Mach number vs. temperature (h), seen in polar coordinates. The color maping is such that sub-(super)sonic regions appear in blue (red). The vortex center is always located at $\varphi=0$. The dashed black lines indicate $v_{\varphi}=v_{\mathrm{K}}$. The global structure is not self-similar when $h$ varies, as one can see the Keplerian line undergoes a reconnection as temperature increases, and spirals in the outer disk are shocking (Mach 1, white) closer to the vortex. Small supersonic (red) regions are found in the inner region of the disk ( $r \simeq 120 \mathrm{AU})$, as is highlighted in an inset in the rightmost panel. The flow remains subsonic everywhere else.

density waves in a Keplerian disk. Such spiral waves would have the same frequency as the Rossby wave creating the vortex.

As opposed to companion-excited spirals, those are not caused by gravitational interaction and are observed in massless disks simulations such as ours (Huang et al. 2019). For Rossby vortices, the launching point is radially close to the vorticity extremum and the spiral corotates with the vortex. As a consequence, for spiral arms with different launching points, the RWI explanation may be safely rejected.

However, it must be noted that the apparent launching point of the spiral, that is, the origin of its detectable part, graphically indicated as a blue hatched mark, departs from its physical origin, namely the eye of the vortex. For instance, Fig. 9 shows a $\sim 90^{\circ}$ discrepancy between the actual launching point and the apparent origin of the main spiral arm. The figure also shows that, considering only spectral resolution as an experimental limitation, plane-RWI spirals are detectable as soon as the sensitivity is sufficient to resolve the bulk signature of the vortex. In short, spirals produce projected velocities just marginally smaller than the vortex's bulk. We further note that plane-RWI spirals are a pure tracer of radial velocities $v_{r}$, which are observationally characterized by a change of sign in projected velocities across the major axis.

We note that the pitch angle of the spiral increases with $h$, as a consequence of a higher sound speed. Hence, radial velocities are not self-similar across our models, as hotter disks produce higher Mach numbers, as illustrated in Fig. 12.

\section{Discussion}

\subsection{Numerical versus practical differences}

In Sect. 3.1, we showed that a promising data reduction strategy for vortex dynamic extraction in sharp density jumps was to 


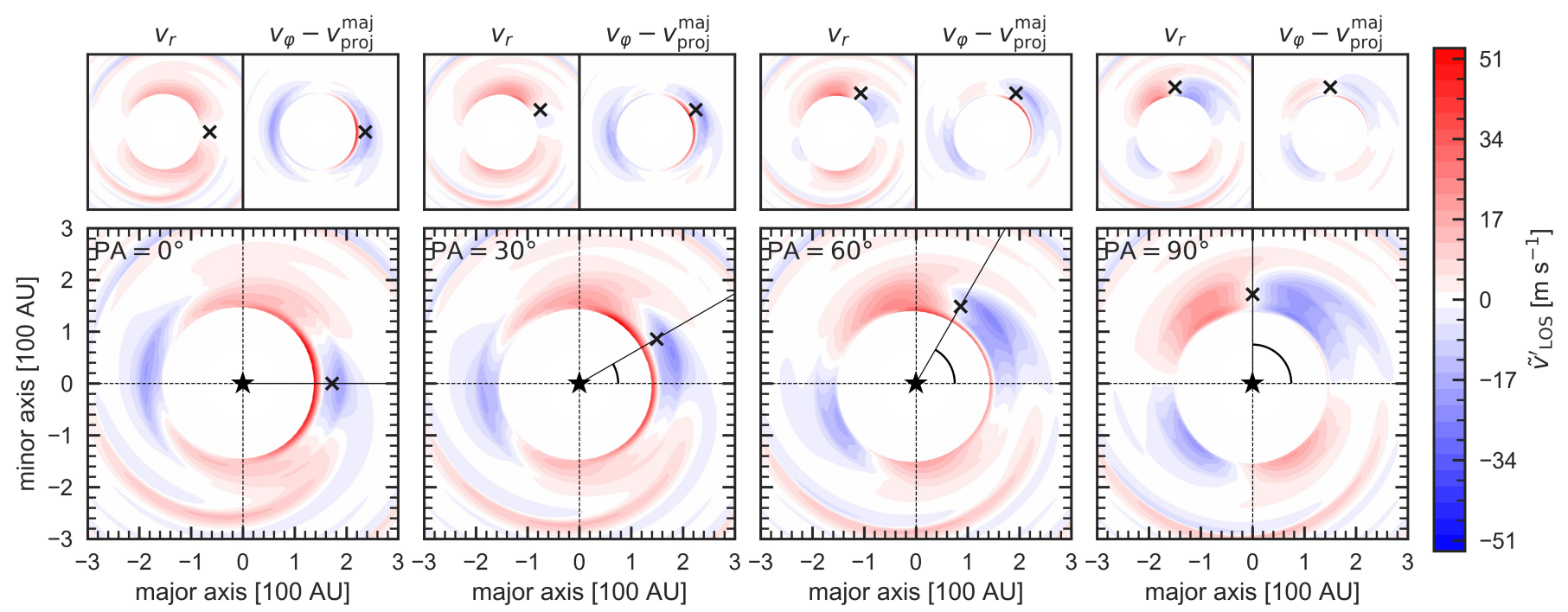

Fig. 13. Practical application of our data reduction method. The figure is similar to Fig. 8 except that $v_{\text {proj }}^{\text {maj }}$ is subtracted instead of $\left\langle v_{\varphi}\right\rangle$.
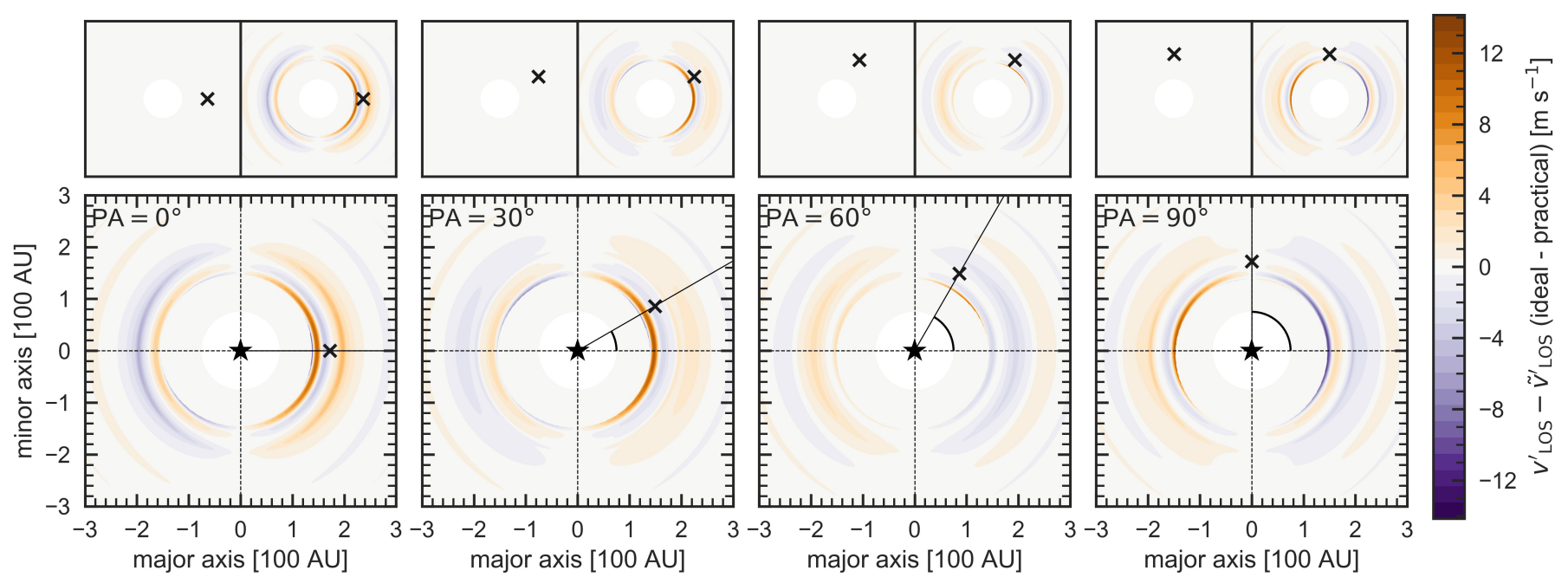

Fig. 14. Difference between numerical (Fig. 8) and practical (Fig. 13) cases. By construction, $v_{\text {LOS }}^{\prime}-\tilde{v}_{\text {LOS }}^{\prime}$ is a separable function err $(r, \varphi)=$ $f(r) \cos (\varphi)$ where the density mask is axisymmetric.

subtract $\left\langle v_{\varphi}\right\rangle$, and that the projected velocity seen on the major axis ( $v_{\text {proj }}^{\text {maj }}$ for shorts) gives a reasonable proxy for it. In order to test the error implied by this approximation, this strategy is applied in Fig. 13. Consistently with our previous estimation, this more realistic view shows very little difference to the first, idealized estimation (Fig. 8). Figure 14 quantifies that 2D discrepancy as a difference between the numerical and practical cases. We find the discrepancy to reach at most $\sim 7 \mathrm{~m} \mathrm{~s}^{-1}$.

\subsection{Spiral detection}

As shown in Sect. 3.4, the projected velocities seen in spiral arms are comparable in amplitude to those attained by the core of the vortex core. However, angular resolution might constitute an additional limitation to identify those secondary structures. In Fig. 15 we simulate a limited angular resolution via Gaussian kernel convolution to the simulated velocity map, where the mean component of azimuthal velocity $\left\langle v_{\varphi}\right\rangle$ is subtracted prior to projection. We observe that the contrast sharpness of the main spiral pattern is altered but not destroyed by limited spatial resolution alone. We note that the spiral arm appears marginally broader in Fig. 13 as compared with the numerical case Fig. 8 . The velocity flip pattern however remains visible and is unaltered by the limited spatial resolution.

\subsection{Origin of the cavity in $H D 142527$}

The most up-to-date simulations for the thermal emission of HD 142527 were performed in smooth-particle hydro (SPH) by Astropy Collaboration (2018) and do not feature vortex formation. This study was focused on explaining as many features as possible with the excitation provided by the eccentric stellar companion. However, it must be noted than SPH solvers generate numerical viscosities $\sim 10^{-2}$ (Arena \& Gonzalez 2013), which are much greater than typical values used in RWI vortex studies ${ }^{3}$ (Lyra et al. 2009; Hammer et al. 2017, 2019; Ono et al. 2016), so this possibility was inherently not included in their study. In

\footnotetext{
3 The model used in this paper is inviscid. Insights into our evaluation of numerical viscosity are given in Appendix B.
} 


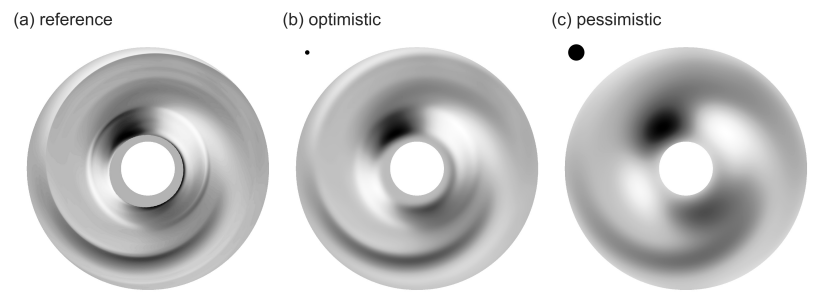

Fig. 15. Qualitative comparison between a simulation-precision velocity map (left), and against artificially lowered spatial resolution, simulation with a Gaussian kernel convolution. Kernels with angular size (in proportions of the target) $7 \%$ (center), and a 3 times larger one $21 \%$ (right) are shown. With the distance of HD 142527, the center panel corresponds to the recent high resolution obtained by Keppler et al. (2019). No noise is added. Projected velocities are shown in linear gray-scale, where $v_{\text {LOS }}^{\prime}<0$ is shown in light gray and $v_{\text {LOS }}^{\prime}>0$ shown in is dark gray. Secondary spiral patterns are lost at low resolution but the primary remains visible. Beam size is shown as a black dot. The velocity map corresponds to the rightmost panel in Fig. 9.

the present work, we remained agnostic regarding how the initial unstable density jump was formed. The stellar companion, while not included in our model, provides a plausible cause to the cavity. However, gravitational perturber-induced Rossby vortices have been studied in the context of circular orbital motion (Li et al. 2005). How eccentricity and inclination in the orbit of the companion affects the formation of vortices, within an appropriately inviscid medium, remains to be studied.

\subsection{Limits of this approach}

An important limitation of the model is the lack of a vertical dimension. In a more realistic context, plane velocities $\left(v_{r}, v_{\varphi}\right)$ are only detectable if the disk is inclined, which in turn affects measurements by line-of-sight integration. This effect would however be mitigated by choosing optically thick molecular lines. Moreover, Méheut et al. (2012b) showed that 3D vortices have a non-negligible vertical velocity component while they form (typically $10 \%$ of the characteristic azimuthal velocity signature). As the RWI growth time is typically shorter than the vortex lifetime by one or two orders of magnitude, it seems reasonable to neglect vertical circulation.

It has been shown that the contribution of the disk to the gravitational potential, promotes disk eccentricity (Regály \& Vorobyov 2017b), which in turn amplifies the proper velocity of the vortex. Because this effect is neglected in our model, we expect the resulting velocities to be slightly underestimated in this work.

\section{Conclusions}

We showed that in cavity-hosting circumstellar disks, large eddies produce dynamical signatures on the verge of detectability for current facilities.

As the dynamical imprint of a vortex resides in the nonaxisymmetric part of the velocity field, it is crucial to the detection to be able to subtract the axisymmetric component from observations. In the case of a vortex formed at the inner edge of a cavity-hosting disk, a Keplerian power law is not a correct proxy for the mean azimuthal velocity. This is because pressure gradients prone to vortex formation imply large deviations from Keplerian velocities. Nevertheless, as projected velocities of the observational major axis directly map the azimuthal motion, a better mask can be obtained by averaging both sides of the velocity profile on this axis. This approach proved to produce small errors when compared with the actual azimuthal mean component of velocity $\left\langle v_{\varphi}\right\rangle$. We also observed a saturation in the amplitude of projected velocities as the temperature is increased. This result is to be taken with a grain of salt and may point to a limitation of the model we used. Using this amplitude as an estimator for spectral resolution requirement, we conclude that detection of a single large eddy is achievable under a 50$150 \mathrm{~m} \mathrm{~s}^{-1}$ resolution, while the current maximal resolution with ALMA is $\sim 30 \mathrm{~m} \mathrm{~s}^{-1}$ We stress that those minimal requirements were obtained within the particular case of the HD 142527 target, with a relatively low inclination $\left(27^{\circ}\right)$. Minimal resolution would be amplified by a factor $150 \%$ for a more likely, mean inclination of $45^{\circ}$, ceteris paribus. This demanding requirement may explain the current difficulty elucidate in elucidating the nature of known dust clumps in cavity-hosting disks, yet this is achievable with existing facilities. Vortex-free mechanisms could also explain their formation, although observational constraints for fine gas dynamics are needed to properly discriminate between concurrent scenarios. Full 3D modeling would naturally extend the present work, and allow the study of second order effects in line-of-sight integration.

Acknowledgements. C.M.T.R.'s Ph.D. grant is part of ANR number ANR16-CE31-0013 (Planet-Forming-Disks). Computations were performed with OCCIGEN CINES (allocation DARI A0060402231), and "Mésocentre SIGAMM", hosted by Observatoire de la Côte d'Azur. The data presented in this work were proceeded and plotted with Python's data science ecosystem scipy, numpy (van der Walt et al. 2011), matplotlib (Hunter 2007), pandas (McKinney 2010) as well as astropy (Astropy Collaboration 2013, 2018) and the yt framework (Turk et al. 2011). C.M.T. is thankful to Aurélien Crida and Elena Lega for their early proofreading. The authors wish to thank the referee, Zsolt Regàly, for helping in improving the quality of the paper.

\section{References}

Adams, F. C., \& Watkins, R. 1995, ApJ, 451, 314

Andrews, S. M., Terrell, M., Tripathi, A., et al. 2018, ApJ, 865, 157

Arena, S. E., \& Gonzalez, J.-F. 2013, MNRAS, 433, 98

Astropy Collaboration (Robitaille, T. P., et al.) 2013, A\&A, 558, A33

Astropy Collaboration (Price-Whelan, A. M., et al.) 2018, AJ, 156, 123

Ataiee, S., Pinilla, P., Zsom, A., et al. 2013, A\&A, 553, L3

Ataiee, S., Dullemond, C. P., Kley, W., Regály, Z., \& Meheut, H. 2014, A\&A, 572, A61

Avenhaus, H., Quanz, S. P., Schmid, H. M., et al. 2014, ApJ, 781, 87

Barge, P., \& Sommeria, J. 1995, A\&A, 295, L1

Baruteau, C., Barraza, M., Pérez, S., et al. 2019, MNRAS, 486, 304

Benisty, M., Juhasz, A., Facchini, S., et al. 2018, A\&A, 619, A171

Biller, B., Lacour, S., Juhasz, A., et al. 2012, ApJ, 753, L38

Birnstiel, T., Dullemond, C. P., \& Pinilla, P. 2013, A\&A, 550, L8

Boehler, Y., Weaver, E., Isella, A., et al. 2017, ApJ, 840, 60

Bracco, A., Chavanis, P. H., Provenzale, A., \& Spiegel, E. A. 1999, Phys. Fluids, 11,2280

Casassus, S., \& Pérez, S. 2019, ApJ, 883, L41

Casassus, S., Perez M., S., Jordán, A., et al. 2012, ApJ, 754, L31

Casassus, S., Wright, C., Marino, S., et al. 2015a, ApJ, 812, 126

Casassus, S., Marino, S., Pérez, S., et al. 2015b, ApJ, 811, 92

Cazzoletti, P., van Dishoeck, E. F., Pinilla, P., et al. 2018, A\&A, 619, A161

Casassus, S., Marino, S., Lyra, W., et al. 2019, MNRAS, 483, 3278

Chiang, E., \& Youdin, A. 2010, Ann. Rev. Earth Planet. Sci., 38, 493

Christiaens, V., Casassus, S., Perez, S., van der Plas, G., \& Ménard, F. 2014, ApJ, $785, \mathrm{~L} 12$

de Val-Borro, M., Edgar, R. G., Artymowicz, P., et al. 2006, MNRAS, 370, 529

de Val-Borro, M., Artymowicz, P., D’Angelo, G., \& Peplinski, A. 2007, A\&A, 471, 1043

Dong, R., Liu, S.-Y., Eisner, J., et al. 2018, ApJ, 860, 124

Fu, W., Li, H., Lubow, S., \& Li, S. 2014, ApJ, 788, L41

Fukagawa, M., Tamura, M., Itoh, Y., et al. 2006, ApJ, 636, L153 
Fukagawa, M., Tsukagoshi, T., Momose, M., et al. 2013, Publ Astron Soc Jpn Nihon Tenmon Gakkai, 65

Gaia Collaboration (Brown, A. G. A., et al.) 2016, A\&A, A595

Goldreich, P., \& Tremaine, S. 1979, ApJ, 233, 857

Hammer, M., Kratter, K. M., \& Lin, M.-K. 2017, MNRAS, 466, 3533

Hammer, M., Pinilla, P., Kratter, K. M., \& Lin, M.-K. 2019, MNRAS, 482, 3609

Harten, A., Lax, P. D., \& van. Leer, B. 1983, SIAM Rev., 25, 35

Huang, P., Dong, R., Li, H., Li, S., \& Ji, J. 2019, ApJ, 883, L39

Hunter, J. D. 2007, Comput. Sci. Eng., 9,99

Isella, A., Huang, J., Andrews, S. M., et al. 2018, ApJ, 869, L49

Kataoka, A., Tsukagoshi, T., Momose, M., et al. 2016, ApJ, 831, L12

Keppler, M., Teague, R., Bae, J., et al. 2019, A\&A, 625, A118

Koren, B. 1993, Numerical Methods for Advection-Diffusion Problems (Braunschwelg, Wlesbaden: Vieweg), 117

Lai, D., \& Tsang, D. 2009, MNRAS, 393, 979

Li, H., Finn, J. M., Lovelace, R. V. E., \& Colgate, S. A. 2000, ApJ, 533, 1023

Li, H., Colgate, S. A., Wendroff, B., \& Liska, R. 2001, ApJ, 551, 874

Li, H., Li, S., Koller, J., et al. 2005, ApJ, 624, 1003

Lin, M. 2012, AGU Fall Meeting Abstracts, 21, P2 1B

Lovelace, R. V. E., Li, H., Colgate, S. A., \& Nelson, A. F. 1999, ApJ, 513, 805

Lyra, W., Johansen, A., Klahr, H., \& Piskunov, N. 2009, A\&A, 493, 1125

McKinney, W. 2010, in Proceedings of the 9th Python in Science Conference, 51

McNally, C. P., Nelson, R. P., \& Paardekooper, S.-J. 2018, MNRAS, 477, 4596

Méheut, H., Keppens, R., Casse, F., \& Benz, W. 2012a, A\&A, 542, A9

Méheut, H., Yu, C., \& Lai, D. 2012b, MNRAS, 422, 2399

Montesinos, M., Perez, S., Casassus, S., et al. 2016, ApJ, 823, L8

Muto, T., Grady, C. A., Hashimoto, J., et al. 2012, ApJ, 748, L22

Ono, T., Muto, T., Takeuchi, T., \& Nomura, H. 2016, ApJ, 823, 84

Perez, S., Casassus, S., Ménard, F., et al. 2015, ApJ, 798, 85

Pérez, S., Casassus, S., Hales, A., et al. 2020, ApJ, 889, L24
Pineda, J. E., Szulágyi, J., Quanz, S. P., et al. 2019, ApJ, 871, 48 Pinte, C., Price, D. J., Ménard, F., et al. 2018, ApJ, 860, L13

Pinte, C., van der Plas, G., Ménard, F., et al. 2019, Nat. Astron., 3, 1109 Porth, O., Xia, C., Hendrix, T., Moschou, S. P., \& Keppens, R. 2014, ApJS, 214, 4

Price, D. J., Cuello, N., Pinte, C., et al. 2018, MNRAS, 477, 1270 Rafikov, R. R. 2002, ApJ, 572, 566

Rameau, J., Chauvin, G., Lagrange, A.-M., et al. 2012, A\&A, 546, A24

Rayleigh, J. W. 1879, Proc. Lond. Math. Soc., 1, 57

Regály, Z., \& Vorobyov, E. 2017a, MNRAS, 471, 2204

Regály, Z., \& Vorobyov, E. 2017b, A\&A, 601, A24

Regály, Z., Juhasz, A., Sándor, Z., \& Dullemond, C. P. 2012, MNRAS, 419, 1701

Regály, Z., Sándor, Z., Csomós, P., \& Ataiee, S. 2013, MNRAS, 433, 2626

Richard, S., Barge, P., \& Le Dizès, S. 2013, A\&A, 559, A30

Shakura, N. I., \& Sunyaev, R. A. 1973, A\&A, 500, 33

Tanga, P., Babiano, A., \& Dubrulle, B. 1996, Icarus, 121, 158

Teague, R., \& Foreman-Mackey, D. 2018, Res. Notes Am. Astron. Soc., 2, 173

Teague, R., Guilloteau, S., Semenov, D., et al. 2016, A\&A, 592, A49

Teague, R., Bae, J., Bergin, E. A., Birnstiel, T., \& Foreman-Mackey, D. 2018a, ApJ, 860, L12

Teague, R., Bae, J., Birnstiel, T., \& Bergin, E. A. 2018b, ApJ, 868, 113

Turk, M. J., Smith, B. D., Oishi, J. S., et al. 2011, ApJS., 192, 9

van der Marel, N., van Dishoeck, E. F., Bruderer, S., et al. 2016, A\&A, 585, A58

van der Walt, S., Colbert, S. C., \& Varoquaux, G. 2011, Comput. Sci. Eng., 13, 22

Verhoeff, A. P., Min, M., Pantin, E., et al. 2011, A\&A, 528, A91

Xia, C., Teunissen, J., Mellah, I. E., Chané, E., \& Keppens, R. 2018, ApJS, 234 30

Yen, H.-W., Liu, H. B., Gu, P.-G., et al. 2016, ApJ, 820, L25

Zhu, Z., \& Baruteau, C. 2016, MNRAS, 458, 3918

Zhu, Z., \& Stone, J. M. 2014, ApJ, 795, 53 


\section{Appendix A: Evaluations of aspect ratios}

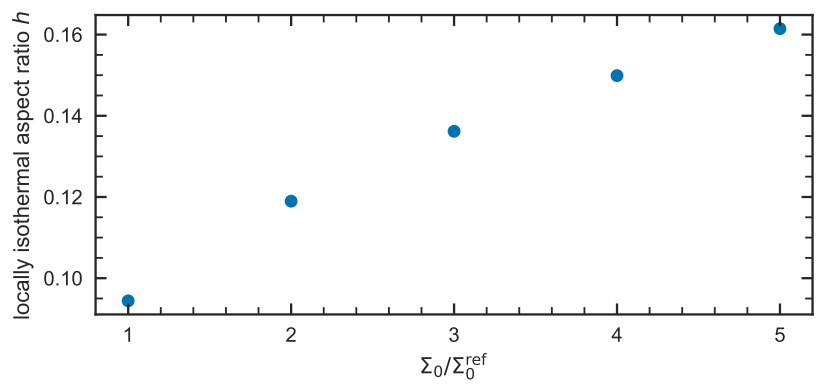

Fig. A.1. Correspondence between locally isothermal aspect ratio and "disk mass" in our simulations. The leftmost model is the reference.

\section{A.1. Equivalence to locally isothermal}

Our model differs from the widely used locally isothermal prescription in that it is not defined in terms of scale height

$H=h r\left(r / r_{*}\right)^{\beta}$,

where $h$ is the disk aspect ratio and $\beta$ is the flaring. We can nonetheless draw an equivalence with those parameters for the power-law density distribution at the core of Eq. (3), such that $\Sigma(r)=\Sigma_{0}\left(r / r_{*}\right)^{-1}$. In the locally isothermal prescription, the scale height is usually defined such that $H^{2}=c_{\mathrm{s}}^{2} / \Omega_{K}^{2}$, so we can equate this with Eq. (A.1) to get

$$
\begin{aligned}
h^{2} r^{2}\left(r / r_{*}\right)^{2 \beta} & =\frac{\gamma p / \Sigma}{G M / r^{3}} \\
& =\frac{\gamma S}{G M} r^{3} \Sigma^{\gamma-1} \\
& =\frac{\gamma S \Sigma_{0}^{\gamma-1}}{G M} r^{3}\left(r / r_{\mathrm{j}}\right)^{1-\gamma},
\end{aligned}
$$

at which point we deduce an effective aspect ratio and disk flaring, in terms of the actual simulation parameters

$\left\{\begin{array}{l}h^{2}=\frac{\gamma S \Sigma_{0}^{\gamma-1} r_{*}}{G M}, \\ \beta=1-\gamma / 2=1 / 6 .\end{array}\right.$

We note that our fixed resolution corresponds to $\Delta r / H\left(r_{\mathrm{j}}\right) \simeq$ 0.04 for the reference model. Figure A.1 shows the resulting variation in $h$ as we scale up $\Sigma_{0}$, following Eq. (A.3).

\section{A.2. Spiral fitting}

In Fig. 9, we fitted the linear-regime spiral wave shape (Goldreich \& Tremaine 1979; Rafikov 2002; Muto et al. 2012) given by

$\varphi(r)=\varphi_{o}-\frac{\operatorname{sgn}\left(r-r_{o}\right)}{H_{o}}$
$\times\left(\left(r / r_{o}\right)^{1+\beta}\left[\frac{1}{1+\beta}-\frac{1}{1-\alpha+\beta}\left(r / r_{o}\right)^{-\alpha}\right]-\left[\frac{1}{1+\beta}-\frac{1}{1-\alpha+\beta}\right]\right)$,

where $\alpha, \beta$ are power-law exponents defined as $\Omega \propto r^{-\alpha}$ and $c_{\mathrm{S}} \propto r^{-\beta}$, respectively. The quantities $\left(r_{o}, \varphi_{o}\right)$ are the coordinates of the spiral origin, while $H_{o}$ is a scale height at this position.

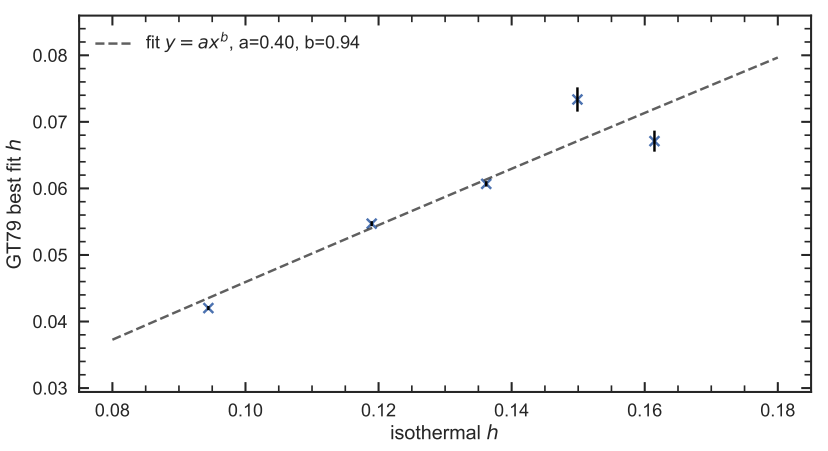

Fig. A.2. Aspect ratios as defined in Appendix A.1 vs. empirical values obtained from fitting Eq. (A.4). The latter is roughly $40 \%$ of the former.

The fit was performed with $H_{o}$ as a free parameter, so we the corresponding aspect ratio, differs from the locally isothermal equivalent $h$ value used throughout the paper and described in the previous section. Figure A. 2 shows values against each other.

\section{Appendix B: Numerical viscosity evaluation}
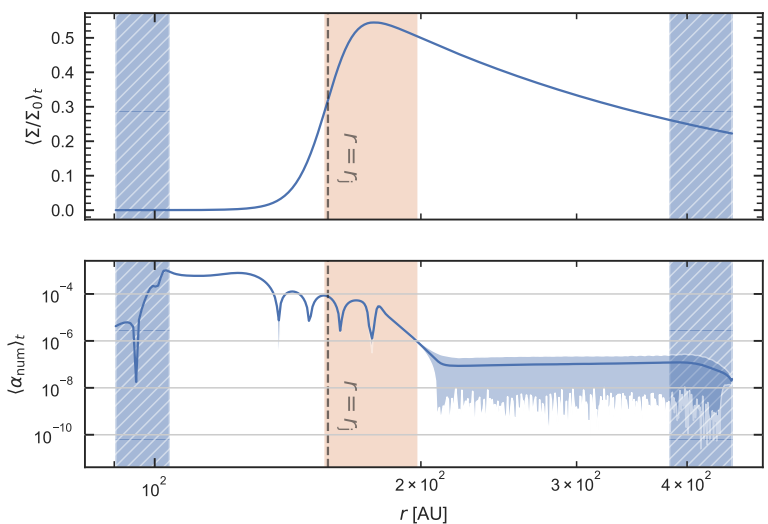

Fig. B.1. Density (top) and numerical viscosity equivalent $\alpha$ value (bottom) time-averaged over 10 orbital periods $\left(t / t_{*} \in[90,100]\right)$ with a sampling rate of 0.1 orbital periods. The solid blue shadows indicate the variation interval over the sample time series, showing that the profile is very stable in the region of interest. The hatched regions highlight the wave-killing zones, while the orange region loosely indicates the vortex-forming region, spanning one scale height away from the local density maximum.

In order to estimate numerical viscosity $v_{\text {num }}(r)$, performed a 1D in a $1 \mathrm{D}$ run, with identical parameterization as our reference $2 \mathrm{D}$ run (run1). The analytical initial conditions constitute a stable equilibrium since RWI cannot grow in 1D. Since our boundary conditions do not impose mass flux, any radial mass transport $\dot{M}$ through the simulation domain is caused by numerical viscosity such that $v_{\text {num }} \Sigma=|\dot{M}| / 3 \pi$. In terms of the Shakura \& Sunyaev (1973) alpha viscosity model $v_{\text {num }}=\alpha_{\text {num }} H c_{\text {s }}$, thus finally

$\alpha_{\text {num }}=\frac{2}{3}\left|\frac{v_{r}}{h c_{\mathrm{s}}}\right|$.

The obtained profile, time-averaged, is plotted in Fig. B.1. The highest numerical viscosities $\left(\alpha_{\text {num }} \sim 2 \times 10^{-3}\right)$ are reached in the cavity, while $\alpha_{\text {num }}$ stays bounded $<10^{-4}$ in the vortex-forming region, roughly represented in orange. 